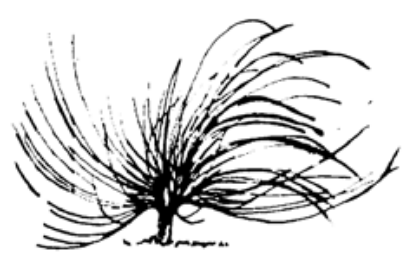

\title{
Evaluación de desempeño académico: La competencia léxica productiva como competencia con validez predictiva
}

\author{
Martín Gonzalo Zapico ${ }^{1}$ \\ Universidad Nacional de Mar del Plata \\ Instituto Nacional de Formación Docente Continua \\ Mar del Plata, Buenos Aires, Argentina \\ athenspierre@gmail.com
}

\begin{abstract}
Resumen
El desempeño académico en el nivel superior, específicamente la posibilidad de predecirlo, es un tema de enorme profusión en términos tanto de teoría como de práctica. Hay un acuerdo entre quienes investigan de que, dentro de lo difícil que es establecer factores predictivos, tres tienen un grado moderado, pero no concluyente de correlación: el desempeño previo, la motivación y el juicio crítico. La hipótesis de este trabajo es que el conocimiento léxico productivo de un sujeto, que puede ser evaluado en términos de sinonimia, es un factor que determinará el rendimiento académico. Para dicho propósito se realizó una comparación cuantitativa en el desempeño obtenido
\end{abstract}

\section{(1) (1) \& 89}

Recibido: 21 de mayo de 2016-Aprobado: 22 de setiembre de 2016

1 Martín Gonzalo Zapico es Profesor en Letras recibido en la Universidad Nacional de Mar del Plata. Actualmente se desempeña como docente-investigador en las cátedras de Lengua I y Lengua y Literatura y su Didáctica I. Ha publicado variedades de artículos en las áreas de lingüística, educación y psicología, y asistido a numerosos congresos de dichas disciplinas. 
por un grupo de estudiantes de la materia Didáctica de la Lengüa y la Literatura del Profesorado en Letras del IFDC, a los cuales previamente se les había administrado un test de sinónimos. Los resultados sugieren que el conocimiento léxico productivo, evaluado en términos de sinonimia, es un factor que permite predecir el rendimiento académico.

Palabras clave: Evaluación, desempeño académico, vocabulario productivo.

\begin{abstract}
Academic performance in higher education, specifically the ability to predict it, is an issue of enormous profusion in terms of both theory and practice. There is an agreement among researchers on how difficult it is to establish predictors, three have a moderate but inconclusive correlation degree: past performance, motivation and judgment. The hypothesis of this paper is that the productive lexical knowledge of a subject, which can be evaluated in terms of synonymy, is a factor that will determine academic performance. For this purpose, a quantitative comparison of the performance obtained by a group of students of Teaching of Language and Literature Teacher from the Language Professors of the IFDC, whom previously had been administered a test of synonyms, was performed. The results suggest that productive lexical knowledge, assessed in terms of synonymy, is a factor that predicts academic performance.
\end{abstract}

Keywords: evaluation, academic performance, productive lexical

\title{
Introducción
}

La siguiente investigación surge en el marco del Instituto Nacional de Formación Docente Continua (Argentina, San Luis) como proyecto orientado a la mejora de la calidad educativa en términos de formación del profesorado, para lo cual se busca obtener recursos basados en investigaciones radicadas en la propia institución. 
En este marco, la propuesta de buscar factores de rendimiento en términos de predicción surge como respuesta a una situación que, si bien sucede en la institución, es una tendencia a nivel nacional y cada vez se profundiza más: la alta tasa de fracaso y deserción en los niveles de educación superior. Más allá de las hipótesis que pueden establecerse para explicar este fenómeno, resulta más interesante y operativo observar y analizar cuáles son los factores que parecen orientar un buen rendimiento en términos de éxito (aprobar las diversas instancias de evaluación) para proponer algún lineamiento que ayude a mejorar el desempeño general del alumnado.

Así, la experiencia como investigador y docente, sumada a las observaciones de colegas que se desempeñan en materias de primer año, me llevó a investigar las competencias de lecto-escritura más generales, puesto que la mayor cantidad de reclamos por parte del personal docente es que el alumnado llega al nivel superior sin competencias léxicas básicas. La sinonimia fue elegida como parámetro a partir de la necesidad de emplear un test sencillo, pero contundente en términos de conocimiento de la lengua, atendiendo al hecho de que el lenguaje orienta asuntos como el pensamiento, la comunicación y es una herramienta fundamental para afrontar una carrera de nivel superior.

\section{Estado del arte}

Hablar de desempeño académico es hablar de un tema absolutamente complejo tanto en términos de problemáticas que comprende la deserción en los distintos niveles escolares (Rodríguez, Pita y Torrado, 2004; Tejedor y Repiso, 2007; Vélez, Schiefelbein y Valenzuela, 1994), la tasa de finalización de carrera (Díaz y Blanco, 2016), los problemas en torno a la motivación (Pozo, Álvarez, Contreras y Reséndiz, 2008), las relaciones entre desempeño y contexto (Meerbeke y González, 2005), definiciones sobre lo que es evaluar y qué es el desempeño -las distintas formas de evaluación (Arribas, 2012), las críticas a la evaluación (Méndez 2001; Pérez, 2001), etc.-, precisar qué es un buen desempeño o uno malo (Navarro, 2003), metodologías de investigación -estudios cuantitativos longitudinales (Jiménez, Izquierdo y Blanco, 2000), estudios de cohorte (Kohan, Solano y Renault, 2001), seguimiento, análisis de entrevistas y percepciones (Vargas, 2007), etc.- y hasta campos de 
aplicación (investigar para desarrollar políticas educativas, para sanear una situación concreta, para obtener un panorama de la cuestión, etc).

Por eso, ante todo, es pertinente acotar qué es exactamente aquello que estamos investigando: la posibilidad de predecir el desempeño en términos de éxito académico a partir de conocer la competencia léxica productiva de un sujeto. Diversos estudios (Benitez, Giménez y Osicka, 2000; Carvajal, 2013, Lozano y Lozano, 2015; Garaigordobil y Torres, 1996; Lugo, Villatoro, Medina-Mora y García, 1996; Pérez, 2002; Pozo, Álvarez, Contreras y Reséndiz, 2008) muestran que son fundamentalmente tres los parámetros que tienen una alta tasa de fiabilidad para predecir el desmpeño académico: el desempeño previo (estudiantes que en el nivel secundario han tenido una alta tasa de éxito y han concluido con un buen promedio suelen sostener el rendimiento $\mathrm{y}$ adaptarse sin problemas al primer año de una carrera), la motivación (estudiantes que estudian carreras que han elegido como primera opción, con altas expectativas sobre el futuro y optimismo, con baja ansiedad, suelen tener alta tasa de éxito en el primer año) y el juicio crítico (estudiantes que puntúan alto en los test de pensamiento crítico, es decir, que poseen una capacidad de comprensión lógica alta, suelen tener un buen desempeño).

Aún así, en esas mismas investigaciones se aclara que otros estudios han realizado investigaciones donde muestran resultados que parecen contradecir lo planteado en el párrafo anterior. Esto pone de manifiesto que los tres parámetros señalados no son suficientemente fiables como para hablar de una correlación alta entre ellos y el desmempeño académico.

La idea de que el conocimiento léxico podría llegar a perfilarse como un predictor fiable del desempeño académico surge a partir de investigaciones previas (especialmente Zapico, 2016) donde se mostró la importancia de las competencias generales para llevar adelante un buen rendimiento. El vocabulario como gran constructo puede ser analizado en dos planos diferentes: el de la recepción y el de la producción. El de la recepción tiene que ver con las competencias de un sujeto para interpretar un texto codificado mediante un sistema simbólico cualquiera (en lo que compete a educación, el idioma en el cual se comunican docente-alumnado así como de los textos) a partir del reconocimiento de los relaciones sintagmáticas y paradigmáticas fundamentales que rige la gramática de una lengua. Por otro lado, el vocabulaio productivo se 
refiere a la capacidad de un sujeto de producir enunciados que se adecuen a las normas de una lengua (en este ámbito entran la redacción, la capacidad de expresión verbal, la argumentación, entre otras). Si bien es cierto que en todo proceso comunicativo se requieren ambas, diversos test (Casso 2010) ponen de manifiesto que el vocabulario productivo susbsume al recepctivo, es decir, que es más fácil para un hablante o incluso un aprendiz leer un texto que producirlo.

Estrictamente, el conocimiento léxico en términos de vocabulario productivo como competencia general (Casso, 2010) puede ser comprendido en términos de sinonimia a partir de investigaciones sobre la distancia semántica (Zapico y Vivas, 2014; Zapico y Vivas, 2015 y Zapico, 2015) donde se observó que a mayor indiferenciación de los significados (mayor grado de sinonimia) hay una mayor capacidad léxico-productiva en el sujeto, puesto que es capaz de dar una mayor cantidad de descriptores para conceptos similares lo que pone, así, de relieve las diferencias de significado.

De la unión de los dos grandes conceptos (la predicción del desmepeño y la competencia léxica) es que se postula la hipótesis de que un mayor grado de competencia léxica debería redundar en un mayor desempeño, puesto que se estaría en posesión de capacidades de comprensión, análisis, síntesis y comunicación, que son necesarias para el afrontamiento de cualquier texto o trabajo.

Para poner a prueba dicha hipótesis se llevó a cabo un estudio cuantitativo de carácter correlacional-descriptivo, puesto que se busca observar posibles correlaciones entre dos variables: competencia léxico-productiva (evaluada en términos de sinonimia) y desempeño académico; a partir de la hipótesis de que ambas varían en un sentido positivo (a mayor grado de competencia léxico-productiva mayor desempeño académico).

\section{Metodología}

La investigación se dividió en dos partes. En la primera se obtuvo información sobre la competencia léxico-productiva de los sujetos de la muestra en cuestión. En la segunda se observó el rendimiento académico de dichos sujetos a la luz de las puntuaciones obtenidas.

Materiales: Para la elaboración del Z-Test de sinónimos se tomaron las Normas de producción de atributos semánticos en castellano 
rioplatense (Vivas, Comesaña, García Coni, Vivas y Yerro, 2013), a partir de las cuales se obtuvieron 80 palabras de alta frecuencia que poseen sinónimos según WordReference diccionario de sinónimos (2016). Luego, a través de la comparación con la base CREA de las 1000 palabras más frecuentes de la Real Academia Española (2016) y el juicio de pares especialistas en el área, se llegó a una lista final de 40 palabras. La prueba consiste en dar al menos un sinónimo de cada palabra y emplearla en una oración gramaticalmente correcta donde la palabra y su sinónimo sean intercambiables. Por cada acierto se suma un punto, con un mínimo de 0 y un máximo de 40. Para el análisis de datos se utilizó el software de análisis Statistic Pack for Social Sciences (SPSS).

Muestra: 40 estudiantes que cursaron la materia Didáctica de la Lengua y la Literatura en el año 2015, proporción de hombres y mujeres balanceada entre 18 y 30 años de edad, del Profesorado de Lengua y Literatura del Instituto de Formación Docente Continua.

Procedimiento: Una vez obtenido el consentimiento oral que fue posteriormente respaldado por su versión escrita, se procedió a la administración del Z-Test. Este se llevó a cabo de manera individual en el espacio de trabajo del docente-investigador, en condiciones óptimas de iluminación y sonido, sin distractor alguno. El tiempo de administración fue de treinta (30) minutos, aunque ningún sujeto superó dicha medida.

A partir del conjunto inicial de 40 sujetos y sus puntuaciones en el test de sinónimos se delimitaron cuatro grupos: A( $>30$ Alta competencia léxica) $\mathrm{B}(20>30$ Buena competencia léxica) $\mathrm{C}(10>20$ Regular competencia léxica) y $\mathrm{D}(<10$ Baja competencia). Una vez definidos los grupos se observó su desmpeño en dos evaluaciones escritas (EE1 y EE2) y una evaluación oral (EO)

Análisis de datos: Se realizó una comparación de éxito en el curso a través de la comparación porcentual de estudiantes en un sentido general (la aprobación o no de la curso) y particular (las tres instancias evaluativas). Además se realizó una comparación de medida de tendencia central (media) para los resultados obtenidos en: EE1, EE2 y EO, correlacionadas con los grupos A,B,C y D de desenvolvimiento léxico. 


\section{Resultados y discusión}

A continuación se muestran y discuten los resultados de la experiencia, empezando por el éxito en en el curso de los grupos A,B,C y D.

\section{ÉXITO EN EL CURSO}

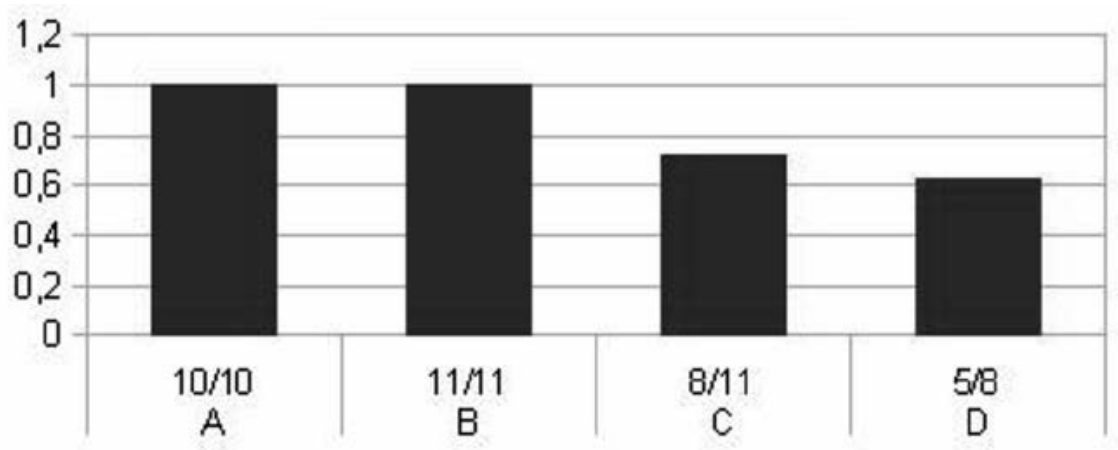

Figura 1. Diferencias entre estudiantes de los distintos grupos conformados respecto a la finalización del curso.

En la figura 1 se observa el porcentaje de éxito en el curso para cada uno de los grupos. En el caso del grupo A y B, los grupos de mayor desenvolvimiento léxico, un $100 \%$ de quienes empezaron completaronel curso. La baja en el éxito se registra a partir del grupo C, desenvolvimiento regular, con un $72 \%$ y registra su número más bajo en el D donde solo concluyeron un $62 \%$. Esto parece indicar que hay una correlación entre el desempeño académico y el grado de competencia léxica en el alumnado. La inferencia está construida a partir de la intuición de que la realización de prácticos, la resolución de consignas de diversa índole y todos los procesos de organización de ideas a través del lenguaje requieren competencias óptimas de léxico productivo, lo cual da una amplia ventaja a los grupos A y B que, si bien no registran diferencia entre sí, marcan una diferencia notable con los otros dos grupos donde empiezan a marcarse los problemas para relacionarse con la planificación, organización y producción de textos académicos como respuesta a diferentes consignas. Parece ser que un mayor dominio del léxico permite afrontar, de manera más idonea, los desafíos que supone un curso de nivel superior, perfilando sujetos activos, que no solo son 
capaces de resignificar el saber, sino que también gozan de una mayor competencia comunicativa, lo que les permite un mejor intercambio con sus docentes y con sus propios compañeros y compañeras, lo cual permite transitar el curso de manera más amena.

A continuación, se muestra la figura 2 que compara el desempeño de los grupos A,B,C y D por las medias de los resultados obtenidos en las distintas instancias evaluativas, así como una evaluación final que corresponde al promedio de las tres medias, calificadas a partir del sistema numérico estandar del 0 al 10 donde la nota máxima posible en 10 (diez) y la nota de aprobación es 4 (cuatro).

\section{Medias para cada evaluación según grupos}

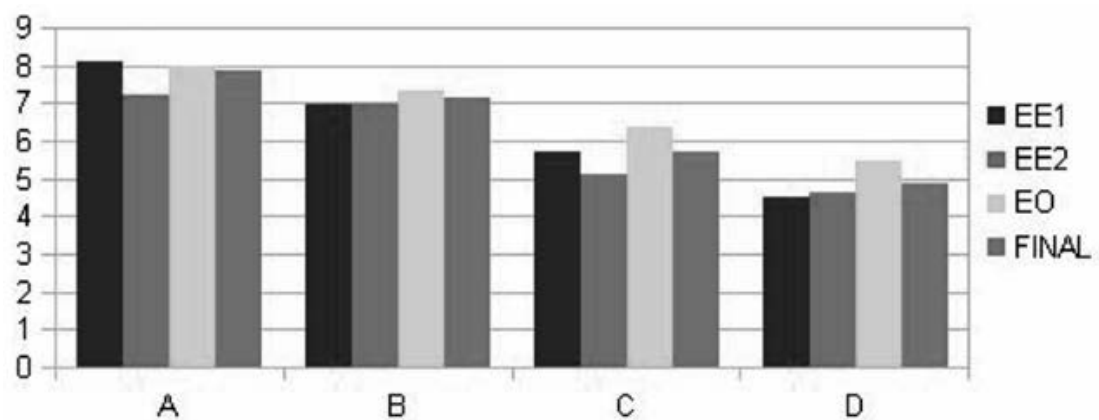

Figura 2. Diferencias entre las medias obtenidas en cada una de las instancias evaluativas para el alumnado de los diferentes grupos.

La primera observación que es llamativa y complementa la figura 1 es que a mayor competencia léxica no solo se observa una mayor taza de finalización de cursada, sino un mayor desempeño en términos de rendimiento en cada una de las evaluaciones. Si antes no parecía haber diferencia entre los grupos A y B, aquí empieza a hacerse notar las diferencias, que son marcadas tanto en las dos instancias escritas $(8,1$ y 7,2 del grupo A contra 7 y 7 del grupo B) como en la oral (8 contra 7,36). Las diferencias porcentuales, que en términos absolutos no parecen tan grandes, deben ser vistas a la luz de que cualquier mejoría en el desempeño de un individuo indica no solo un mejor dominio de los contenidos, sino también de las competencias necesarias para cualquier materia en el marco de la carrera: capacidad de relacionar conceptos, 
capacidad de articular ideas en un texto coherente que dé respuesta a lo que se solicita, etc.

La evidente baja en el desempeño de manera escalonada en función de la competencia léxica da indicios de cuán importante podría llegar a ser la competencia léxica productiva que un sujeto posee antes de entrar a cursar estudios superiores, puesto que estas diferencias se sostienen no solo en las instancias de evaluación escrita sino también oral, manifestando diferencias realmente notables si contraponemos las medias del grupo A $(8,1 / 7,2 / 8)$ contra las medias del grupo D $(4,5 / 4,67 / 5,5)$.

Finalmente, se presentan la tabla 1 con las diferencias de media, desviación estándar, valor mínimo y máximo para los cuatro grupos en cada una de las instancias.

\section{Tabla 1}

Media, desviación estandar, varianza, mayor y menor valor para cada una de las instancias de evaluación y promedio final en cada uno de los grupos

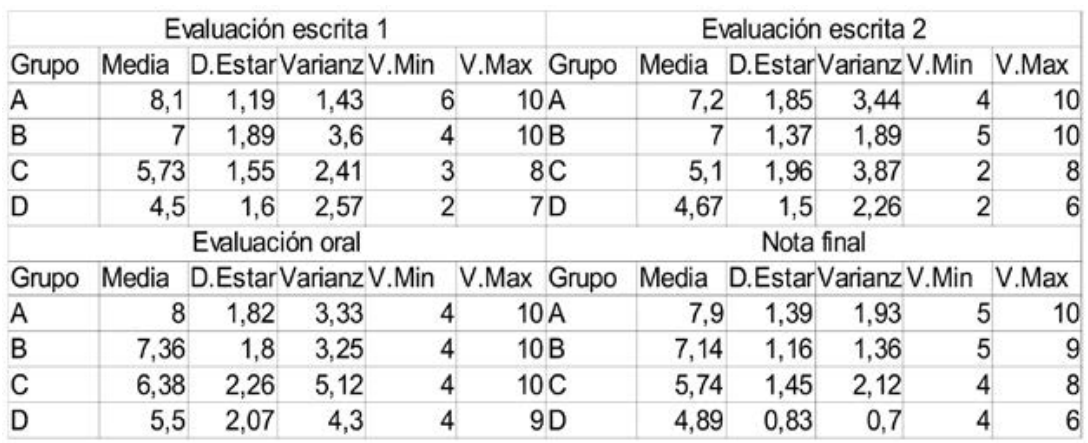

Al observar los números mostrados en la tabla 1 podemos analizar que la mayor varianza y diferencia entre $\mathrm{min} / \mathrm{max}$ sucede en la instancia de evaluación oral, lo cual nos indica que hay al menos una singularidad en cada uno de los grupos. Esto puede explicarse en función del tamaño de cada grupo y teniendo en cuenta que estas singularidades no afectaron el desempeño general respecto a la hipótesis principal, estos datos refuerzan aún más el enunciado principal.

Además, puede observarse una relación directamente proporcional en los valores máximos y mínimos donde, a mayor competencia 
léxica, los valores máximos son más altos y, a menor competencia léxica, los valores son más bajos, no sucediendo nunca que el valor máximo de una categoría inferior supere al de una superior, ni viceversa (nunca el valor mínimo de una categoría de mayor competencia léxica es inferior al de una categoría de menor competencia). En función de las diferencias de las medias A-B y C-D puede observarse una clara diferencia entre ambos subgrupos, que se confirma en los valores mínimos y máximos que son prácticamente indiferenciados entre A-B y C-D, pero marcando entre ellos una gran diferencias poniendo como posible punto de inflexión en el conocimiento léxico productivo un valor cercano a los $18>25$ donde el desempeño aumenta o disminuye de manera significativa de acuerdo a si se está por debajo o por encima de dicho intervalo hipotético.

\section{Conclusión}

El análisis de los datos pone de manifiesto una correlación entre una mayor capacidad léxica productiva y el desempeño académico. Esto puede ser debido a que el proceso de enseñanza-aprendizaje está atravesado y determinado por el lenguaje, en tanto proceso de comunicación en el cual el contenido es el medio a través del cual dialogan docente y alumnado. En esta misma línea es pertinente hacer una aclaración sobre nuestra postura respecto a la importancia del lenguaje: no solo es el medio a través del cual suceden la mayoría de los procesos de enseñanza-aprendizaje en el nivel superior, sino que también es la forma en que tenemos de interactuar con el mundo, decir lo que sentimos, pensar a la otra persona y pensarnos; en fin, entender que la promoción del léxico no solo ayudará en términos académicos, sino en términos también de calidad de vida.

Además, esta investigación apoya una tesis que se sotiene hace años: la enseñanza por competencias no solo es más adaptativa respecto al medio social en el cual los sujetos se desenvuelven, sino también en el mismo medio académico que, poco a poco, debe transformarse para estar a la altura de una realidad compleja, multiforme y en constante cambio. La competencia lingüistica es un tipo de competencia general que se muestra idónea a la hora de afrontar una carrera de nivel superior, por lo cual deberían implementarse políticas en el secundario o en los ingresos a carrera, con el fin de promover un conocimiento de la 
lengua más profundo que ayudará al alumnado a transitar las materias y sus requisitos.

Puede afirmarse que mayormente se cumplió el objetivo de la investigación, y la hipótesis se vio confirmada parcialmente: parece haber una relación entre el desempeño académico en términos de éxito y la competencia léxico-productiva. Esto debería llevarnos a reflexionar sobre la importancia de la enseñanza del léxico, la argumentación y la expresión escrita como prácticas de gran importancia para la formación de sujetos críticos y pensantes, capaces de afrontar las dificultades de una carrera de nivel superior.

No obstante, hay que aclarar que este estudio es de carácter exploratorio en el sentido de que la muestra no es lo suficientemente grande y está marcadamente sectorizada en el campo de las letras. En futuros estudios se empleará el mismo modelo aplicado a diferentes poblaciones universitarias de diferentes características (otras carreras de ciencias humanas e incluso carreras de ciencias exactas y naturales), con el propósito de eliminar variables que podrían alterar el rendimiento en las instancias evaluativas como: los factores socio-económicos, emocionales o singularidades. De esta manera se espera configurar y posicionar esta variable (la competencia léxico-productiva) como un predictor fiable del desempeño académico, lo cual permitirá el diseño, desarrollo e implementación de acciones educativas a efectos de mejorar las competencias básicas del estudiantado en todos los niveles educativos.

\section{Referencias}

Álvarez Mendez, J. (2001). Evaluar para conocer, examinar para excluir. Madrid: Morata.

Arias, J. (2016). La evaluación del rendimiento inmediato en la enseñanza universitaria. Revista Educación, 320, 353-377.

Arribas, J. (2012). El rendimiento académico en función del sistema de evaluación empleado. Revista RELIEVE. 18(1), 1-15. Recuperado de http://www.uv.es/RELIEVE/v18n1/RELIEVEv18n1_3.html

Benitez, M., Gimenez, M. y Osicka, R. (2000). Las asignaturas pendientes y el rendimiento académico. ¿Existe alguna relación? Revista Educación, 189, 224-235

Casso, J. (2010). Análisis y revisión críticas de los materiales de evaluación de la competencia léxica. Elaboración de un test de 
vocabulario de nivel umbral. España. Nebrija: Departamento de Lenguas Aplicadas.

Garaigordobil, M. y Torres, E. (1996). Evaluación de la creatividad en sus correlatos con inteligencia y rendimiento académica. Revista de Psicología Universitas Tarraconesis, 18(1), 87-98

Jiménez, V., Izquierdo, J. y Blanco, A. (2000). La predicción del rendimiento académico: regresión lineal versus regresión logística. Revista Psicothema, 12(2), 248-252.

Lugo, E. Villatoro, J. Medina-Mora, E. y García, F. (1996). Autopercepción del rendimiento académico en estudiantes méxicanos. Revista Méxicana de Psicología, 13(1), 37-47.

Pérez, E. (2002). Validación de características al ingreso como predictores del rendimiento académico en la carrera de medicina. Revista Cubana de Educación Superior, 16(1), 5-18.

Pozo, M. y Álvarez, O. (2007). Desempeño académico de universitarios en relación con ansiedad escolar y auto-evaluación. Revista Acta Colombiana de Psicología, 11(1), 13-23.

RAE. (2016). Diccionario www.rae.es

Rodriguez, S. Fita, E. y Mercedes, T. (2004). El rendimiento académico en la transición secundaria-universidad. Revista de Educación, 334, 391-414.

Meerbeke, A. y González, C. (2005). Factores asociados al rendimiento académico en estudiantes de medicina. Revista Educación Médica, 8(2), 74-82.

Navarro, R. (2003). El rendimiento académico: Concepto, investigación y desarrollo. REICE, 1(2), 1-15. Recuperado de http://www. ice.deusto.es/RINACE/reice/vol1n2/Edel.pdf

Pérez, M. (2001). La evaluación del aprendizaje: Tendencias y reflexión crítica. Revista Cubana de Eduación Superior, 15(1), 85-96.

Renault, G. Kohan, N. y Solano, A. (2001). Factores que intervienen en el rendimiento académico de los estudiantes de psicología y psicopedagogía. Revista Signos Universitarios, 1, 27-34.

Tejedor, F. y Repiso, A. (2007). Causas del bajo rendimiento del estudiante universitario (en opinión de los profesores y alumnos). Propuestas de mejora en el marco del EEES. Revista de Educación, 342, 443-473. 
Vargas, G. (2007). Factores asociados al rendimiento académico en estudiantes universitarios, una reflexión desde la calidad de la educación superior pública. Revista Educación, 31(1), 43-63.

Vélez, E. Schiefelbein, E. y Valenzuela, J. (1994). Factores que afectan el rendimiento académico en la educación primaria. Revista latinoamericana de innovaciones educativas en Argentina, 17, 1-16.

Vivas, J., Comesaña, A., García Coni, A., Vivas, L., Yerro, M. (2013). Distribución de los atributos semánticos en función del tipo de categoría y campo semántico. Resultados preliminares para la confección de normas de atributos. En M. C. Richaud y V. Lemos (Comp.), Psicología y otras ciencias del comportamiento. Compendio de investigaciones actuales I (pp. 311-333). Libertador San Martin: Editorial Universidad Adventista del Plata.

Word Reference. (2014). Diccionario de sinónimos. www.wordreference.com/es

Zapico, G. (2016). Evaluación de estrategias de enseñanza orientadas a competencias: Una comparación de caso entre estilo orientado a la competencia y el orientado al contenido. Revista Ensayos Pedagógicos (En Prensa).

Zapico, G. y Vivas, J. (2015). La sinonimmia desde una perspectiva lingüistico-cognitiva. Medición de la distancia semántica. Onomázein. 32, 198-211. DOI:10.7764/onomázein.32.11

Zapico, G. (2015). Análisis de las distancias semánticas entre conceptos. La sinonimia . Revista Psiciencia doi: 10.5872/psiencia/7.1.041011

Zapico, G. y Vivas, J. (2014). La sinonimia como caso particular de distancia semántica. Encontros Bibli, 19(40), 253-256. 